\title{
Morphological, Structural, and Electrical Characterization of Sol-Gel-Synthesized ZnO Nanorods
}

\author{
M. Kashif, ${ }^{1}$ U. Hashim, ${ }^{1}$ M. E. Ali, ${ }^{2}$ K. L. Foo, ${ }^{1}$ and Syed M. Usman Ali ${ }^{3}$ \\ ${ }^{1}$ Nano Biochip Research Group, Institute of Nano Electronic Engineering (INEE), Universiti Malaysia Perlis (UniMAP), \\ 01000 Kangar, Perlis, Malaysia \\ ${ }^{2}$ Nanotechnology and Catalysis Research Centre, Universiti Malaya, 50603 Kuala Lumpur, Malaysia \\ ${ }^{3}$ Department of Electronic Engineering, NED University of Engineering and Technology, Karachi 75270, Pakistan
}

Correspondence should be addressed to M. Kashif; kashif_bme@yahoo.com

Received 18 November 2012; Accepted 3 February 2013

Academic Editor: Ping Xiao

Copyright (C) 2013 M. Kashif et al. This is an open access article distributed under the Creative Commons Attribution License, which permits unrestricted use, distribution, and reproduction in any medium, provided the original work is properly cited.

$\mathrm{ZnO}$ nanorods were grown on thermally oxidized p-type silicon substrate using sol-gel method. The SEM image revealed highdensity, well-aligned, and perpendicular $\mathrm{ZnO}$ nanorods on the oxidized silicon substrate. The XRD profile confirmed the $c$-axis orientation of the nanorods. PL measurements showed the synthesized $\mathrm{ZnO}$ nanorods have strong ultraviolet (UV) emission. The electrical characterization was performed using interdigitated silver electrodes to investigate the stability in the current flow of the fabricated device under different ultraviolet (UV) exposure times. It was notified that a stable current flow was observed after $60 \mathrm{~min}$ of UV exposure. The determination of stable current flow after UV exposure is necessary for UV-based gas sensing and optoelectronic devices.

\section{Introduction}

Zinc oxide $(\mathrm{ZnO})$ belongs to II-VI semiconductor compound which has a wide bandgap energy $(\sim 3.37 \mathrm{eV})$ and stable wurtzite structure with lattice spacing $a=0.325 \mathrm{~nm}$ and $c=0.521 \mathrm{~nm}$. The direct wide bandgap favours $\mathrm{ZnO}$ for the UV detection applications. Among various types of photodetectors, metal-semiconductor-metal (MSM) photoconductive detectors are generally the simplest in terms of fabrication and also easy to obtain higher gain [1]. The large exciton binding energy $(\sim 60 \mathrm{meV})$ makes $\mathrm{ZnO}$ suitable for optoelectronic applications, especially at temperature near and above room temperature [2]. Zinc oxide has attracted much research attention because of its unique properties and versatile applications in transparent electronics [3], ultraviolet (UV) light emitters [4], and piezoelectric devices [5]. UV photoresponse in $\mathrm{ZnO}$ was first observed by Mollow in 1940s [6]. After his study, many researchers studied the UV region photoresponse by utilizing single crystal, poly-, and crystalline films as well as nanowires [7-9]. A slow response time of UV photodetector based on polycrystalline $\mathrm{ZnO}$ thin films, ranging from a few minutes to several hours, is commonly observed [10-15]. Due to large surface-tovolume ratio and miniaturized dimensionality of the active area, $\mathrm{ZnO}$ nanostructures are expected to have high photon conductance [16]. $\mathrm{ZnO}$ nanostructures can synthesized with different morphologies such as nanowires [17], nanotubes [18], nanorings [19], nano-tetrapods [20], and nanoflakes [21] via variety of methods including MOCVD [22], thermal evaporation [23], molecular beam epitaxy (MBE) [24], electrochemical deposition [25], spray pyrolysis [26], and sol-gel [27]. Among these methods, the sol-gel technique is the simplest and least expensive. One-dimensional $\mathrm{ZnO}$ nanostructures are gaining great attention because of their potential applications in nanoscale electronic and optoelectronic devices [28, 29]. Thus, a well-characterized and easily preparable $\mathrm{ZnO}$ nanostructure has long been expected for its application in specialized optoelectronic devices. Most of the studies on UV sensor have been focused on the mechanism investigation [30,31] and improving the sensitivity [32,33]. However, little attention has been paid to monitor the stability time of the sensor. Keem et al. [34] studied the photocurrent in $\mathrm{ZnO}$ nanowires and tested the sensor after $3000 \mathrm{~s}$ of UV illumination. 
In this study, $\mathrm{ZnO}$ nanorods with photodetector functions were synthesized using an easily performable sol-gel method. The current-to-voltage $(I-V)$ measurements were performed at different time intervals to investigate the effect of timing on the sensor at a fixed UV power.

\section{Experimental}

$\mathrm{ZnO}$ thin films were prepared on a thermally oxidized $\mathrm{p}$ type silicon substrate of resistivity $1-100 \Omega \mathrm{cm}^{-1}$ using a sol-gel method. Before coating the seed layer, the silicon substrate was cleaned with RCA1 and RCA2 [27] to remove the contaminants. RCA1 solution was prepared by mixing DI water, ammonium hydroxide $\left[\mathrm{NH}_{4} \mathrm{OH}(27 \%)\right]$, and hydrogen peroxide $\left[\mathrm{H}_{2} \mathrm{O}_{2}(30 \%)\right]$ by maintaining the ratio of $5: 1: 1$. For the RCA2 preparation, hydrochloric acid [HCL (27\%)] and hydrogen peroxide $\left[\mathrm{H}_{2} \mathrm{O}_{2}(30 \%)\right]$ were mixed in DI water by maintaining the composition at $6: 1: 1$. The residual oxide layer was removed by dipping the substrate into a BOE solution followed by washing with DI water and drying under $\mathrm{N}_{2}$ flow. After the cleaning process, the silicon wafer was thermally oxidized to generate a $1 \mu \mathrm{m}$ layer of $\mathrm{SiO}_{2}$. The oxide layer is used to isolate the deposited $\mathrm{ZnO}$ film from silicon substrate in order to neglect the influence of current flow from silicon substrate. All the chemicals were used without further purification. The seed solution preparation and hydrothermal growth were performed following the procedure described in literatures $[21,27]$. Briefly, 3.84 gm zinc acetate dihydrate was dissolved in $50 \mathrm{~mL}$ 2-methoxyethanol and $1.069 \mathrm{gm}$ monoethanolamine. Monoethanolamine acts as a stabilizer and was added dropwise under stirring and constant temperature. Thus a $\mathrm{ZnO}$ solution of concentration $0.35 \mathrm{M}$ was prepared. The solution was stirred for $2 \mathrm{~h}$ at $60^{\circ} \mathrm{C}$ until a transparent and homogeneous solution was obtained. The prepared solution was stored overnight at room temperature. The aged solution was spin coated on $\mathrm{SiO}_{2}$ grown p-type silicon substrate using spin coater which was rotated at $3000 \mathrm{rpm}$ for $30 \mathrm{~s}$. The film was preheated at $250^{\circ} \mathrm{C}$ for $20 \mathrm{~min}$ after each coating. The coating-to-drying process was repeated for five times and thus produced film was annealed at $550^{\circ} \mathrm{C}$ for $1 \mathrm{hr}$.

The $\mathrm{ZnO}$ nanorods were grown on seeded oxidized $\mathrm{p}$ type silicon substrate using hydrothermal growth method. The growth solution was prepared by mixing zinc nitrate hexahydrate $(25 \mathrm{mM})$ and hexamethyltetramine $(25 \mathrm{mM})$ in $150 \mathrm{~mL}$ deionized water (DI). The seeded substrate was placed vertically so that the seeded side facing down in the growth solution. The growth was done in a preheated oven at $93^{\circ} \mathrm{C}$ for $6 \mathrm{hrs}$. After the growth, sample was washed with DI water to remove the residual salts and dried using $\mathrm{N}_{2}$ gun. The UV detector was fabricated as an MSM configuration using silver as the electrode. Interdigitated Ag electrodes were deposited on the $\mathrm{SiO}_{2} / \mathrm{Si}$ substrate using vacuum thermal evaporator. IDE device with the size of $8.75 \times 5.00 \mathrm{~mm}$ was successfully deposited on the $\mathrm{SiO}_{2} / \mathrm{Si}$ substrate using hard mask. In this work, IDE with 8 fingers was used whereby the width and length of each finger was $0.25 \mathrm{~mm}$ and $4.75 \mathrm{~mm}$, respectively. The spacing between the two fingers was $0.5 \mathrm{~mm}$.

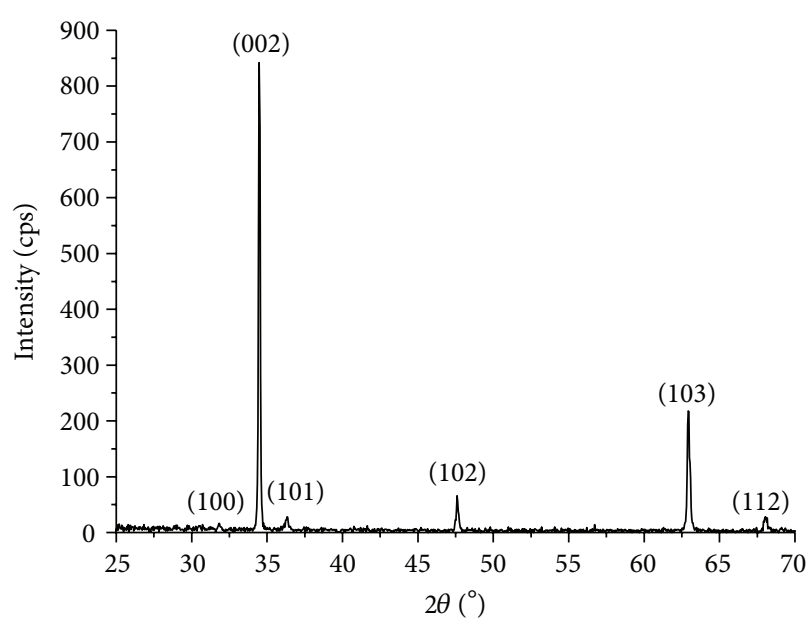

FIGURE 1: XRD pattern of $\mathrm{ZnO}$ nanorods.

The surface morphology and structure of $\mathrm{ZnO}$ nanorods were examined using scanning electron microscopy (SEMJEOL 6460LA). X-ray diffractometer (XRD) from Bruker D8 was used for phase identifications. The PL spectra of the $\mathrm{ZnO}$ nanorods were measured using $\mathrm{He}-\mathrm{Cd}$ laser from Horiba Jyobin (HR 800) with an excitation wavelength of $325 \mathrm{~nm}$ at a power of $20 \mathrm{~mW}$ at room temperature. A UV LED with a wavelength of $365 \mathrm{~nm}$ and power of $2 \mathrm{~mW}$ was used as the illuminating source. A Keithley 2400 sourcemeter was used to measure the $I-V$ characteristics. All the measurements were carried out under ambient environment.

\section{Results and Discussion}

The XRD pattern of the $\mathrm{ZnO}$ nanorods is shown in Figure 1. It consisted of several peaks at (100), (002), (101), (102), (103), and (112) planes. All the diffraction peaks were indexed to the hexagonal structure of the $\mathrm{ZnO}$ according to JCPDS Card no. 036- 1451. The lattice constants " $a$ " and " $c$ " for the $\mathrm{ZnO}$ nanostructures were calculated using the Bragg's law [35] as:

$$
n \lambda=2 d \sin \theta \text {, }
$$

where $n$ is the order of diffraction, $\lambda$ is the X-ray wavelength and $d$ is the spacing between planes of given Miller indices $h$, $k$, and $l$. The plane spacing is related to the lattice constants " $a$ " and " $c$ " and the Miller indices by the following relation [35]:

$$
\frac{1}{d_{(h k l)}^{2}}=\frac{4}{3} \frac{\left(h^{2}+h k+k^{2}\right)}{a^{2}}+\frac{l^{2}}{c^{2}} .
$$

The calculated values for lattice parameters are $a=3.2449 \AA$ and $c=5.196 \AA$, indicating that the products were pure $\mathrm{ZnO}$. The strongest peak (002) at $2 \theta=34.489^{\circ}$ with full-width halfmaximum (FWHM) of $0.132^{\circ}$ showed good quality crystal and $c$-axis orientation. No other peaks characterizing the impurities of the precursors, such as zinc nitrate and hexamethyltetramine, were detected. Table 1 shows the structural 
TABLE 1: The structural parameters of $\mathrm{ZnO}$ nanorods.

\begin{tabular}{lccccccc}
\hline$h k l$ & $2 \theta$ & $\begin{array}{c}d_{\text {calculated }}(\AA) \\
(\AA)\end{array}$ & $\begin{array}{c}d_{\text {JCDS }} \\
(\AA \%\end{array}$ & FWHM & $D$ & $\begin{array}{c}\sigma \\
\left(\mathrm{N} \cdot \mathrm{m}^{-2}\right)\end{array}$ \\
\hline 100 & 31.818 & 2.81018 & 2.8143 & 0.146 & $0.333^{\circ}$ & 235.885 & \\
002 & 34.489 & 2.598 & 2.6033 & 0.203 & $0.132^{\circ}$ & 595.075 & \\
101 & 36.334 & 2.4705 & 2.4759 & 0.2181 & $0.2216^{\circ}$ & 354.467 & $\begin{array}{c}-7.84 \times \\
10^{9}\end{array}$ \\
102 & 47.608 & 1.9085 & 1.9111 & 0.136 & $0.1818^{\circ}$ & 432.068 & \\
103 & 62.933 & 1.4754 & 1.4771 & 0.114 & $0.236^{\circ}$ & 332.83 & \\
112 & 68.059 & 1.3765 & 1.3781 & 0.116 & $0.2908^{\circ}$ & 270.116 & \\
\hline
\end{tabular}

TABle 2: Calculated " $a$ " and " $c$ " lattice constants for the $\mathrm{ZnO}$ nanorods.

\begin{tabular}{cccc}
\hline & $a$ & $c$ & \\
& $(\AA)$ & $(\AA)$ & $a / c$ \\
\hline \multirow{3}{*}{ Lattice constant } & 3.2449, & 5.196, & 0.625, \\
& $3.248[37]$, & $5.204[37]$, & $0.6241[37]$, \\
& $3.249[38]$ & $5.206[38]$ & $0.62408[38]$ \\
\hline
\end{tabular}

parameters of $\mathrm{ZnO}$ nanorods, whereas Table 2 displayed the calculated lattice constant values in comparison with the reported values. The grain size of the $\mathrm{ZnO}$ nanorods was estimated using the Scherer method [36] as follows:

$$
D=\frac{0.9 \lambda}{\beta \cos \theta},
$$

where $D$ is the grain size, $\lambda$ is the wavelength, $\beta$ is the FWHM of the observed peak, and $\theta$ is the diffraction angle. It is well known that several aspects contribute to the strain in the films, like intrinsic strain induced by impurities and defects in the lattice, expansion coefficient mismatch between the film and substrate. The stress of the $\mathrm{ZnO}$ thin films was estimated using the following formula [36]:

$$
\sigma\left(\mathrm{N} / \mathrm{m}^{2}\right)=-453.6 \times 10^{9}\left(\frac{c_{o}-c}{c_{o}}\right),
$$

where $c$ is the lattice constant obtained from the (002) diffraction peak and $c_{0}$ is $5.205 \AA$ for bulk $\mathrm{ZnO}$ [36].

The SEM image of $\mathrm{ZnO}$ nanorods is demonstrated in Figure 2. The SEM study clearly revealed the well-aligned, perpendicular, and high-density $\mathrm{ZnO}$ nanorods on oxidized silicon substrate. The SEM image strongly correlated with the XRD findings.

The photoluminescence spectrum of $\mathrm{ZnO}$ nanorods at room temperature is shown in Figure 3. The photoluminescence spectra demonstrated a strong UV emission at $381.97 \mathrm{~nm}$ and a weak visible emission at $565.94 \mathrm{~nm}$. This UV band was due to the recombination of free excitons through an exciton-exciton collision process. It is suggested that deep level emissions are associated with the singly ionized oxygen vacancy in $\mathrm{ZnO}$ and results from the recombination of a photogenerated hole with the singly ionized charge state of this defect [39]. Usually, the visible emission from $\mathrm{ZnO}$ is attributed to different defects such as oxygen vacancies $\left(V_{\mathrm{O}}\right)$,

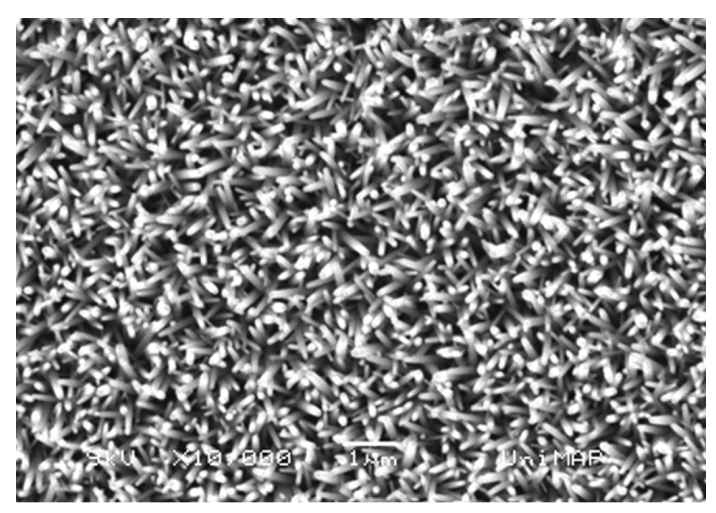

Figure 2: SEM image of $\mathrm{ZnO}$ nanorods.

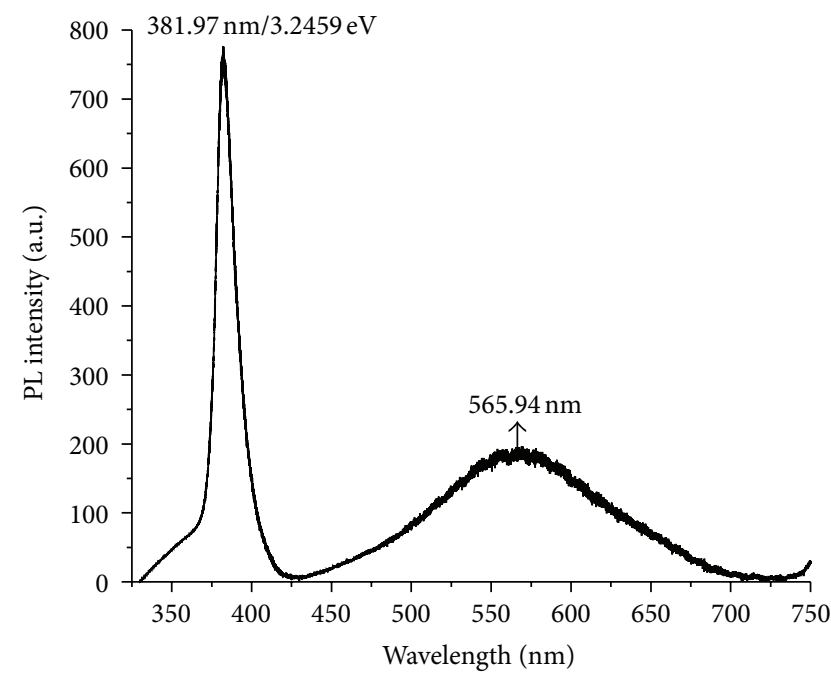

FIgUre 3: Photoluminescence spectra of $\mathrm{ZnO}$ nanorods at room temperature.

zinc vacancies $\left(V_{\mathrm{Zn}}\right)$, or a complex defect involving interstitial zinc $\left(\mathrm{Zn}_{i}\right)$ and interstitial oxygen $\left(\mathrm{O}_{i}\right)[40]$. The low intensity of the visible emission as compared to the near band edge (UV) emission in nanorods was an indication of minimum defect in $\mathrm{ZnO}$ nanorods.

The optical bandgap energy $\left(E_{g}\right)$ of $\mathrm{ZnO}$ nanorods is calculated from photoluminescence spectroscopy using the following relation:

$$
E_{g}=\frac{h C}{\lambda}
$$

where $E_{g}$ is the energy bandgap, $h$ is the Plank's constant, $c$ is the speed of light, and $\lambda$ is the wavelength. By using the above relation the calculated value for the bandgap of obtained $\mathrm{ZnO}$ nanorods was $3.2459 \mathrm{eV}$. Many attempts have been made to relate the refractive index and the energy gap $E_{g}$ through simple relationships [41-46]. However, these relations of $n$ are independent of temperature and incident photon energy. Here the various relations between " $n$ " and " $E_{g}$ " will be reviewed. Ravindra et al. [46] had presented a linear form of "n" as a function of $E_{g}$ :

$$
n=\alpha+\beta E_{g},
$$


TABLE 3: Energy gaps, calculated refractive indices of nanostructure $\mathrm{ZnO}$ using Ravindra et al. [46], Hervé and Vandamme [47], and Ghosh et al. [48] models corresponding to optical dielectric constant.

\begin{tabular}{lcc}
\hline Energy $(\mathrm{eV})$ & $n$ & $\varepsilon_{\infty}$ \\
\hline $3.2459,3.44[49]$, & $2.0355[46], 2.27764$ & $4.143[46], 5.187$ \\
$1.57[50], 0.73[51]$, & {$[47]$,} & {$[47]$,} \\
$0.88[52], 2.26[53]$ & $2.32[48], 2.10[54]$ & $5.38[48]$ \\
\hline
\end{tabular}

where $\alpha=4.048$ and $\beta=-0.62 \mathrm{eV}^{-1}$. Light refraction and dispersion will be inspired. Hervé and Vandamme [47] proposed an empirical relation as follows:

$$
n=\sqrt{1+\left(\frac{A}{E_{g}+B}\right)^{2}},
$$

where $A=13.6 \mathrm{eV}$ and $B=3.4 \mathrm{eV}$. For group-IV semiconductors, Ghosh et al. [48] have published an empirical relationship based on the band structure and quantum dielectric considerations of Penn [55] and van Vechten [56] as:

$$
n^{2}-1=\frac{A}{\left(E_{g}+B\right)^{2}},
$$

where $A=25 E_{g}+212, B=0.21 E_{g}+4.25$, and $\left(E_{g}+B\right)$ refers to an appropriate average energy gap of the material. The calculated refractive indices of the end-point compounds and energy gaps are listed in Table 3, and give a good agreement with experimental $[49,54]$ and theoretical studies [50-53]. This is verified by the calculation of the optical dielectric constant $\varepsilon_{\infty}$ which depends on the refractive index. Note that the optical dielectric constant $\varepsilon_{\infty}=n^{2}$ [57]. It is clear that the investigated refractive index " $n$ " using model of Ravindra et al. [46] is important for nanostructure $\mathrm{ZnO}$ in enhancing the detecting and sensing. It means high absorption may be attributed to increase sensors efficiency.

To investigate the effect of UV exposure time on the sensor, current-to-voltage characteristics are shown in Figure 4. It was observed that with exposure to UV light, the current drastically increased from $4.46 \times 10^{-6}$ A to $2.23 \times 10^{-5} \mathrm{~A}$. However, with the increment of UV exposure time from $30 \mathrm{~min}$ to onward, the changes in current flow gradually became static and no significant changes in current flow were observed after $60 \mathrm{~min}$ of UV exposure as shown in Figure 4(b). The stability in current flow upon UV exposure is important to get a reliable signal in the gas sensing applications. The standard equation for the diode current can be written as [58]:

$$
I=I_{s} \exp ^{(q V / n k T-1)}
$$

where $I_{s}$ is the saturation current, $V$ is the applied voltage, $n$ is the ideality factor, $q$ is the electronic charge, $k$ is the Boltzmann constant, and $T$ is the temperature in Kelvin. The barrier height can be calculated using the following relation [58]:

$$
I_{s}=A A^{*} T^{2} \exp ^{\left(-q \Phi_{B} / k T\right)}
$$

where $A$ is the contact area, $A^{*}$ is the effective Richardson's constant $\left(32 \mathrm{~cm}^{-2} \mathrm{~K}^{-2}\right)$ [59], $T$ is the absolute temperature, $q$ is the electron charge, and $\phi_{B}$ is the barrier height. It was observed that the barrier height decreased from $0.718 \mathrm{eV}$ to $0.65 \mathrm{eV}$ from dark to $65 \mathrm{~min}$ of UV illumination.

Figure 5 shows the $\log (I)$ versus voltage curve of the fabricated sensor with dark and under various UV exposure times. Our $\log (I)$ versus voltage curve followed the same behaviour as reported by $\mathrm{Li}$ et al. [60]. The calculated value for ideality factor was 18 under the dark condition and that value decreased to 9.21 when the sensor was illuminated for $1 \mathrm{~min}$. For the rest of the UV exposure times, there were no significant variations in the ideality factor of the fabricated sensor.

The current versus time relationship as well as the repeatability curve of $\mathrm{ZnO}$ nanorod-based sensor are shown in Figure 6. When the UV light is on, the resistance of the device sharply decreases. This may be ascribed to the surface oxygen adsorption of $\mathrm{ZnO} \mathrm{NWs}$ and the electron-hole pairs generated by UV light in NWs [61]. The repeatability of the device was tested by switching UV light on and off at the same time intervals. A good reproducibility is noted and approximately identical current rise and decay was observed over 3 cycles. This indicated that UV sensing mechanism involves some reversible interactions among $\mathrm{ZnO}$ nanorods, ambient gases, and UV light [61].

Some theoretical and experimental studies suggested that photoresponse of $\mathrm{ZnO}$ is primarily governed by desorption and adsorption of oxygen [61]. Thus the overall photoresponse can be divided into two separate processes: one is a bulk material-related process that is fast and reproducible. The other is a surface-related process that is slow. The latter can be explained in the following way: first, an electron can be trapped by oxygen to form a chemically adsorbed surface state as:

$$
\mathrm{O}_{2} \text { (gas) }+\mathrm{e}^{-} \longrightarrow \mathrm{O}_{2}{ }^{-} \text {(ad) }
$$

When photon energy equal to or higher than the bandgap hits on the $\mathrm{ZnO}$ surface, an electron-hole pair is generated. The positively charged hole neutralizes the chemisorbed oxygen $\left(\mathrm{O}_{2}\right)$ :

$$
h^{+}+\mathrm{O}_{2}^{-}(\mathrm{ad}) \longrightarrow \mathrm{O}_{2} \text { (gas) }
$$

The freed electrons during the photodesorption process contribute to the increase in photoconductivity. The bulkrelated process is the exciton generation by absorption of high-energy photons [62].

\section{Conclusions}

$\mathrm{ZnO}$ nanorods were successfully grown on surface exposed $\mathrm{SiO}_{2}$ of silicon substrate by a sol-gel method. The combined analysis by XRD, SEM, and photoluminescence demonstrated good crystallinity and orientation of the synthesized $\mathrm{ZnO}$ nanorods. The XRD revealed that [002] was the major growth direction of the $\mathrm{ZnO}$ nanorods. The SEM image 


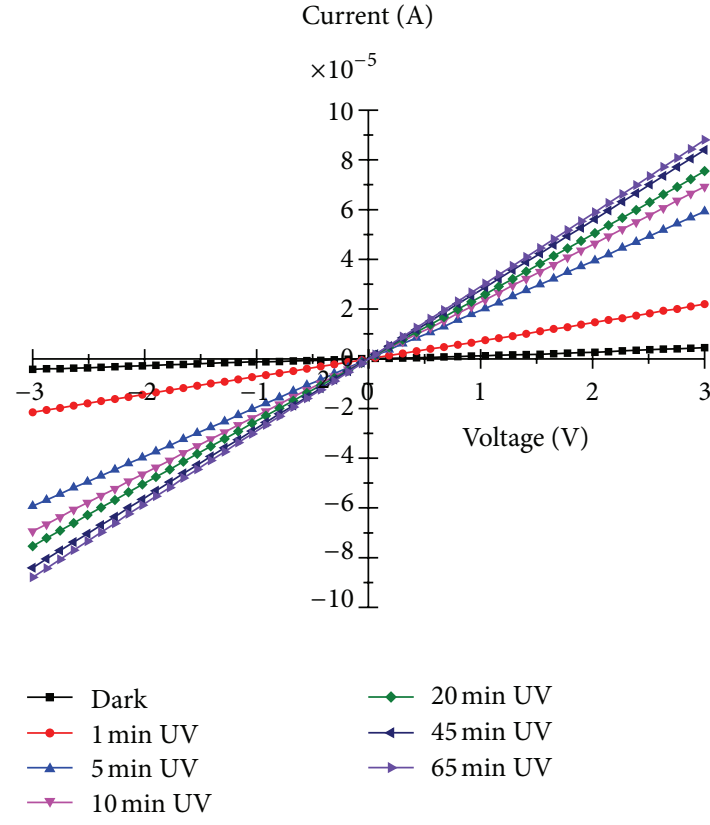

(a)

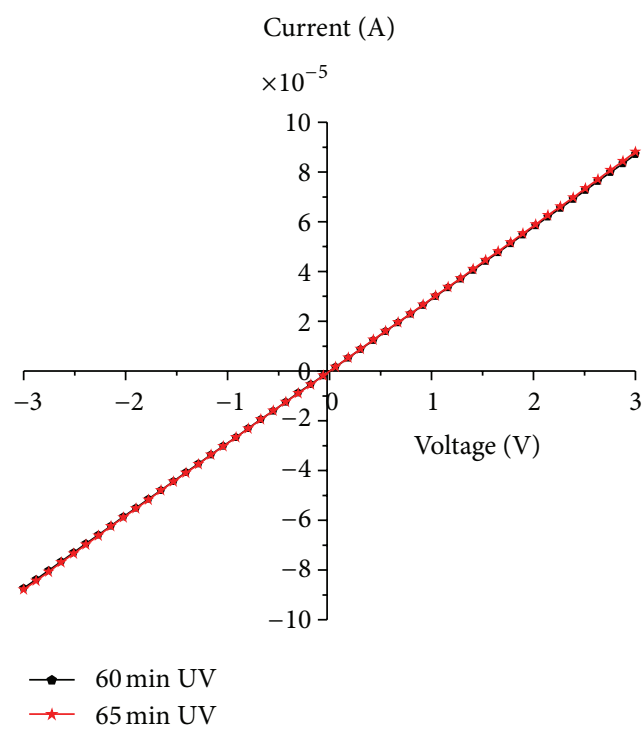

(b)

FIGURE 4: Current to voltage characteristics of $\mathrm{ZnO}$ nanorods under dark condition and different UV exposure times.

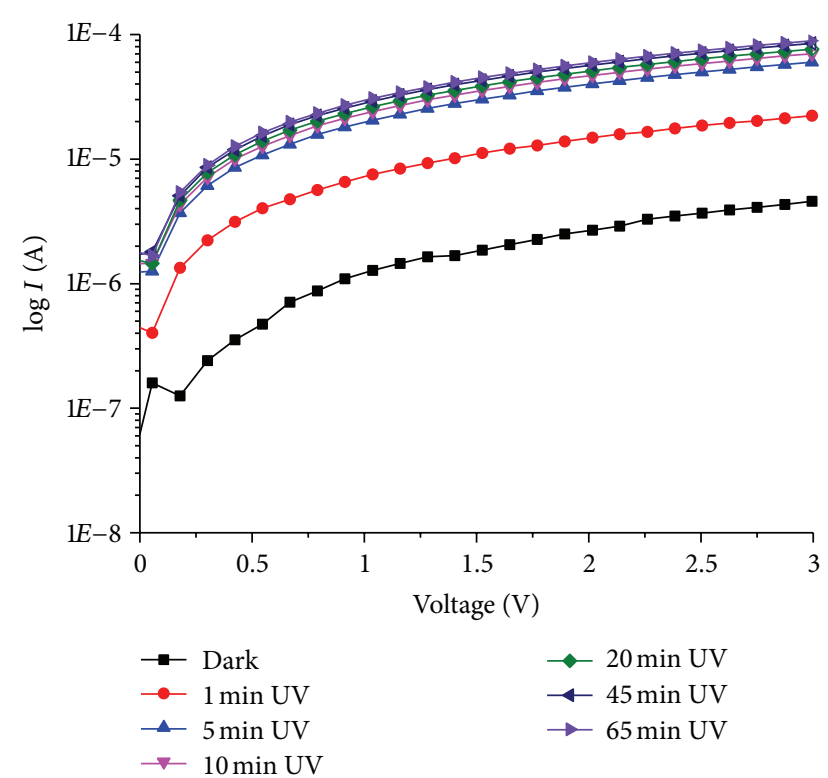

FIGURE 5: $\log (I)$ versus voltage characteristic curves of $\mathrm{ZnO}$ nanorods.

demonstrated that the nanorods are densely populated on the entire silicon substrate. The UV and visible emissions profile of $\mathrm{ZnO}$ nanorods was confirmed by photoluminescence spectra. The current-to-voltage profile showed stability in photocurrent after $60 \mathrm{~min}$ of UV illumination at room temperature, reflecting its potential applications in miniaturized optoelectronic and gas sensing devices.

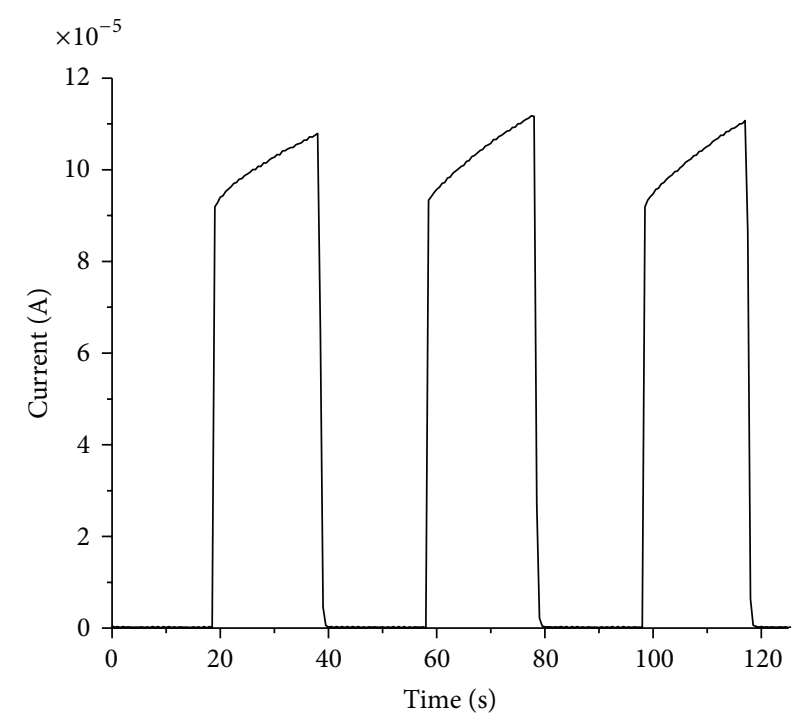

Figure 6: Photocurrent versus time response of $\mathrm{ZnO}$ nanorods at room temperature.

\section{Acknowledgments}

The authors would like to acknowledge the financial support from the FRGS Grant no. 9003-00276 from the Ministry of Higher Education (MOHE). The authors would also like to thank the technical staff of Institute of Nano Electronic Engineering and School of Microelectronic Engineering, Universiti Malaysia Perlis for their kind support to smoothly perform the research. 


\section{References}

[1] J. Sun, Q. Dai, F. Liu et al., "The ultraviolet photoconductive detector based on Al-doped $\mathrm{ZnO}$ thin film with fast response," Science China Physics, Mechanics and Astronomy, vol. 54, no. 1, pp. 102-105, 2011.

[2] W. I. Park, J. S. Kim, G.-C. Yi, M. H. Bae, and H. J. Lee, "Fabrication and electrical characteristics of high-performance $\mathrm{ZnO}$ nanorod field-effect transistors," Applied Physics Letters, vol. 85, no. 21, pp. 5052-5054, 2004.

[3] S. Logothetidis, A. Laskarakis, S. Kassavetis, S. Lousinian, C. Gravalidis, and G. Kiriakidis, "Optical and structural properties of $\mathrm{ZnO}$ for transparent electronics," Thin Solid Films, vol. 516, no. 7, pp. 1345-1349, 2008.

[4] X. Fang, Y. Bando, U. K. Gautam et al., "ZnO and ZnS nanostructures: ultraviolet-light emitters, lasers, and sensors," Critical Reviews in Solid State and Materials Sciences, vol. 34, no. 3-4, pp. 190-223, 2009.

[5] C.-L. Hsu and K.-C. Chen, "Improving piezoelectric nanogenerator comprises $\mathrm{ZnO}$ nanowires by bending the flexible PET substrate at low vibration frequency," The Journal of Physical Chemistry C, vol. 116, no. 16, pp. 9351-9355, 2012.

[6] E. Mollow, Proceedings of the Conference on Photoconductivity, John Wiley \& Sons, New York, NY, USA, 1956.

[7] S. Liang, H. Sheng, Y. Liu, Z. Huo, Y. Lu, and H. Shen, "ZnO Schottky ultraviolet photodetectors," Journal of Crystal Growth, vol. 225, no. 2-4, pp. 110-113, 2001.

[8] C. H. Seager and S. M. Myers, "Quantitative comparisons of dissolved hydrogen density and the electrical and optical properties of ZnO," Journal of Applied Physics, vol. 94, no. 5, pp. 2888-2894, 2003.

[9] T. Koida, S. F. Chichibu, A. Uedono et al., "Correlation between the photoluminescence lifetime and defect density in bulk and epitaxial ZnO," Applied Physics Letters, vol. 82, no. 4, pp. 532534, 2003.

[10] P. Sharma, K. Sreenivas, and K. V. Rao, "Analysis of ultraviolet photoconductivity in $\mathrm{ZnO}$ films prepared by unbalanced magnetron sputtering," Journal of Applied Physics, vol. 93, no. 7, pp. 3963-3970, 2003.

[11] D. Basak, G. Amin, B. Mallik, G. K. Paul, and S. K. Sen, "Photoconductive UV detectors on sol-gel-synthesized $\mathrm{ZnO}$ films," Journal of Crystal Growth, vol. 256, no. 1-2, pp. 73-77, 2003.

[12] Y. Takahashi, M. Kanamori, A. Kondoh, H. Minoura, and Y. Ohya, "Photoconductivity of ultrathin zinc oxide films," Japanese Journal of Applied Physics, vol. 33, pp. 6611-6615, 1994.

[13] T. E. Murphy, K. Moazzami, and J. D. Phillips, "Trap-related photoconductivity in $\mathrm{ZnO}$ epilayers," Journal of Electronic Materials, vol. 35, no. 4, pp. 543-549, 2006.

[14] D. H. Zhang and D. E. Brodie, "Photoresponse of polycrystalline ZnO films deposited by r.f. bias sputtering," Thin Solid Films, vol. 261, no. 1-2, pp. 334-339, 1995.

[15] Q. H. Li, T. Gao, Y. G. Wang, and T. H. Wang, "Adsorption and desorption of oxygen probed from $\mathrm{ZnO}$ nanowire films by photocurrent measurements," Applied Physics Letters, vol. 86, no. 12, Article ID 123117, 3 pages, 2005.

[16] C. Soci, A. Zhang, B. Xiang et al., " $\mathrm{ZnO}$ nanowire UV photodetectors with high internal gain," Nano Letters, vol. 7, no. 4, pp. 1003-1009, 2007.

[17] J. Joo, B. Y. Chow, M. Prakash, E. S. Boyden, and J. M. Jacobson, "Face-selective electrostatic control of hydrothermal zinc oxide nanowire synthesis," Nature Materials, vol. 10, no. 8, pp. 596601, 2011.

[18] C. Xu, B.-S. Kim, J.-H. Lee et al., "Seed-free electrochemical growth of $\mathrm{ZnO}$ nanotube arrays on single-layer graphene," Materials Letters, vol. 72, no. 1, pp. 25-28, 2012.

[19] X. Wu, F. Qu, X. Zhang, W. Cai, and G. Shen, "Fabrication of $\mathrm{ZnO}$ ring-like nanostructures at a moderate temperature via a thermal evaporation process," Journal of Alloys and Compounds, vol. 486, no. 1-2, pp. L13-L16, 2009.

[20] A. Khan, S. N. Khan, and W. M. Jadwisienczak, "One step growth of $\mathrm{ZnO}$ nano-tetrapods by simple thermal evaporation process: structural and optical properties," Science of Advanced Materials, vol. 2, no. 4, pp. 572-577, 2010.

[21] M. Kashif, S. M. Usman Ali, M. E. Ali et al., "Morphological, optical, and Raman characteristics of $\mathrm{ZnO}$ nanoflakes prepared via a sol-gel method," Physica Status Solidi (a), vol. 209, no. 1, pp. 143-147, 2012.

[22] D. A. Lamb and S. J. C. Irvine, "Growth properties of thin film $\mathrm{ZnO}$ deposited by MOCVD with n-butyl alcohol as the oxygen precursor," Journal of Crystal Growth, vol. 273, no. 1-2, pp. 111117, 2004.

[23] O. A. Fouad, A. A. Ismail, Z. I. Zaki, and R. M. Mohamed, "Zinc oxide thin films prepared by thermal evaporation deposition and its photocatalytic activity," Applied Catalysis B, vol. 62, no. 1-2, pp. 144-149, 2006.

[24] A. El-Shaer, A. C. Mofor, A. Bakin, M. Kreye, and A. Waag, "High-quality $\mathrm{ZnO}$ layers grown by MBE on sapphire," Superlattices and Microstructures, vol. 38, no. 4-6, pp. 265-271, 2005.

[25] E. Azaceta, R. Tena-Zaera, R. Marcilla et al., "Electrochemical deposition of $\mathrm{ZnO}$ in a room temperature ionic liquid: 1-Butyl1-methylpyrrolidinium bis(trifluoromethane sulfonyl)imide," Electrochemistry Communications, vol. 11, no. 11, pp. 2184-2186, 2009.

[26] M. Krunks, A. Katerski, T. Dedova, I. Oja Acik, and A. Mere, "Nanostructured solar cell based on spray pyrolysis deposited $\mathrm{ZnO}$ nanorod array," Solar Energy Materials and Solar Cells, vol. 92, no. 9, pp. 1016-1019, 2008.

[27] M. Kashif, U. Hashim, M. E. Ali et al., "Effect of different seed solutions on the morphology and electrooptical properties of ZnO nanorods," Journal of Nanomaterials, vol. 2012, Article ID 452407, 6 pages, 2012.

[28] T. K. Lin, S. J. Chang, Y. K. Su, B. R. Huang, M. Fujita, and Y. Horikoshi, "ZnO MSM photodetectors with Ru contact electrodes," Journal of Crystal Growth, vol. 281, no. 2-4, pp. 513517,2005

[29] M. Kang, J. S. Lee, S. K. Sim et al., "Photocurrent and photoluminescence characteristics of networked GaN nanowires," Japanese Journal of Applied Physics, Part 1, vol. 43, no. 10, pp. 6868-6872, 2004.

[30] S.-E. Ahn, H. J. Ji, K. Kim et al., "Origin of the slow photoresponse in an individual sol-gel synthesized $\mathrm{ZnO}$ nanowire," Applied Physics Letters, vol. 90, no. 15, Article ID 153106, 3 pages, 2007.

[31] Y. Li, F. Della Valle, M. Simonnet, I. Yamada, and J.-J. Delaunay, "Competitive surface effects of oxygen and water on UV photoresponse of $\mathrm{ZnO}$ nanowires," Applied Physics Letters, vol. 94, no. 2, Article ID 023110, 3 pages, 2009.

[32] S. L. Chang, M.-C. Park, Q. Kuang et al., "Giant enhancement in UV response of $\mathrm{ZnO}$ nanobelts by polymer surfacefunctionalization," Journal of the American Chemical Society, vol. 129, no. 40, pp. 12096-12097, 2007. 
[33] J. R. S. Aga, D. Jowhar, A. Ueda et al., "Enhanced photoresponse in $\mathrm{ZnO}$ nanowires decorated with CdTe quantum dot," Applied Physics Letters, vol. 91, no. 23, Article ID 232108, 3 pages, 2007.

[34] K. Keem, H. Kim, G. T. Kim et al., "Photocurrent in $\mathrm{ZnO}$ nanowires grown from Au electrodes," Applied Physics Letters, vol. 84, no. 22, pp. 4376-4378, 2004.

[35] O. Lupan, T. Pauporté, L. Chow et al., "Effects of annealing on properties of $\mathrm{ZnO}$ thin films prepared by electrochemical deposition in chloride medium," Applied Surface Science, vol. 256, no. 6, pp. 1895-1907, 2010.

[36] C. Li, X. C. Li, P. X. Yan et al., "Research on the properties of $\mathrm{ZnO}$ thin films deposited by using filtered cathodic arc plasma technique on glass substrate under different flow rate of $\mathrm{O}_{2}$," Applied Surface Science, vol. 253, no. 8, pp. 4000-4005, 2007.

[37] S. Senthilkumaar, K. Rajendran, S. Banerjee, T. K. Chini, and V. Sengodan, "Influence of Mn doping on the microstructure and optical property of ZnO," Materials Science in Semiconductor Processing, vol. 11, no. 1, pp. 6-12, 2008.

[38] "American society for testing and material, powder diffraction files, joint committee on powder diffraction standards," swarthmore, Philadelphia, Pa, USA, 1996.

[39] S. C. Lyu, Y. Zhang, H. Ruh et al., "Low temperature growth and photoluminescence of well-aligned zinc oxide nanowires," Chemical Physics Letters, vol. 363, no. 1-2, pp. 134-138, 2002.

[40] C. C. Kim, J. H. Je, D. W. Kim, H. K. Baik, S. M. Lee, and P. Ruterana, "Annealing behavior of Pd/GaN (0001) microstructure," Materials Science and Engineering B, vol. 82, no. 1-3, pp. 105-107, 2001.

[41] T. S. Moss, "A relationship between the refractive index and the infra-red threshold of sensitivity for photoconductors," Proceedings to the Physical Society B, vol. 63, no. 3, article 302, pp. 167-176, 1950.

[42] V. P. Gupta and N. M. Ravindra, "Comments on the Moss Formula," Physica Status Solidi (b), vol. 100, no. 2, pp. 715-719, 1980.

[43] Y. Al-Douri, Y. P. Feng, and A. C. H. Huan, "Optical investigations using ultra-soft pseudopotential calculations of $\mathrm{Si}_{0.5} \mathrm{Ge}_{0.5}$ alloy," Solid State Communications, vol. 148, no. 11-12, pp. 521524, 2008.

[44] Y. Al-Douri, A. H. Reshak, H. Baaziz et al., "An ab initio study of the electronic structure and optical properties of CdS1-xTex alloys," Solar Energy, vol. 84, no. 12, pp. 1979-1984, 2010.

[45] P. Hervé and L. K. J. Vandamme, "General relation between refractive index and energy gap in semiconductors," Infrared Physics and Technology, vol. 35, no. 4, pp. 609-615, 1994.

[46] N. M. Ravindra, S. Auluck, and V. K. Srivastava, "On the penn gap in semiconductors," Physica Status Solidi (b), vol. 93, no. 2, pp. K155-K160, 1979.

[47] P. J. L. Hervé and L. K. J. Vandamme, "Empirical temperature dependence of the refractive index of semiconductors," Journal of Applied Physics, vol. 77, no. 10, pp. 5476-5477, 1995.

[48] D. K. Ghosh, L. K. Samanta, and G. C. Bhar, "A simple model for evaluation of refractive indices of some binary and ternary mixed crystals," Infrared Physics, vol. 24, no. 1, pp. 43-47, 1984.

[49] K. Hüemmer, "Interband magnetoreflection of ZnO," Physica Status Solidi (b), vol. 56, no. 1, pp. 249-260, 1973.

[50] Z. Charifi, H. Baaziz, and A. H. Reshak, "Ab-initio investigation of structural, electronic and optical properties for three phases of ZnO compound," Physica Status Solidi (b), vol. 244, no. 9, pp. 3154-3167, 2007.
[51] A. Schleife, F. Fuchs, J. Furthmüller, and F. Bechstedt, "Firstprinciples study of ground- and excited-state properties of $\mathrm{MgO}, \mathrm{ZnO}$, and CdO polymorphs," Physical Review B, vol. 73, no. 24, Article ID 245212, 14 pages, 2006.

[52] Y.-N. Xu and W. Y. Ching, "Electronic, optical, and structural properties of some wurtzite crystals," Physical Review B, vol. 48, no. 7, pp. 4335-4351, 1993.

[53] P. Schröer, P. Krüger, and J. Pollmann, "First-principles calculation of the electronic structure of the wurtzite semiconductors ZnO and ZnS," Physical Review B, vol. 47, no. 12, pp. 6971-6980, 1993.

[54] S. Ilican, M. Caglar, and Y. Caglar, "Sn doping effects on the electro-optical properties of sol gel derived transparent $\mathrm{ZnO}$ films," Applied Surface Science, vol. 256, no. 23, pp. 7204-7210, 2010.

[55] D. R. Penn, "Wave-number-dependent dielectric function of semiconductors," Physical Review, vol. 128, no. 5, pp. 2093-2097, 1962.

[56] J. A. van Vechten, "Quantum dielectric theory of electronegativity in covalent systems. I. Electronic dielectric constant," Physical Review, vol. 182, no. 3, pp. 891-905, 1969.

[57] G. A. Samara, “Temperature and pressure dependences of the dielectric constants of semiconductors," Physical Review B, vol. 27, no. 6, pp. 3494-3505, 1983.

[58] M. Kashif, U. Hashim, M. E. Ali, A. A. Saif, M. U. Syed Ali, and M. Willander, "Structural and impedance spectroscopy study of Al-doped $\mathrm{ZnO}$ nanorods grown by sol-gel method," Microelectronics International, vol. 29, no. 3, pp. 131-135, 2012.

[59] F. E. Jones, B. P. Wood, J. A. Myers, C. Daniels-Hafer, and M. C. Lonergan, "Current transport and the role of barrier inhomogeneities at the high barrier n-InP | poly(pyrrole) interface," Journal of Applied Physics, vol. 86, no. 11, pp. 64316441, 1999.

[60] Y. Li, F. D. Valle, M. Simonnet, I. Yamada, and J.-J. C. Delaunay, "High-performance UV detector made of ultra-long $\mathrm{ZnO}$ bridging," Nanotechnology, vol. 20, no. 4, pp. 045501-045504, 2009.

[61] M. Mehrabian, R. Azimirad, K. Mirabbaszadeh, H. Afarideh, and M. Davoudian, "UV detecting properties of hydrothermal synthesized ZnO nanorods," Physica E, vol. 43, no. 6, pp. 11411145, 2011.

[62] C. Y. Liu, B. P. Zhang, Z. W. Lu et al., "Fabrication and characterization of $\mathrm{ZnO}$ film based UV photodetector," Journal of Materials Science, vol. 20, no. 3, pp. 197-201, 2009. 

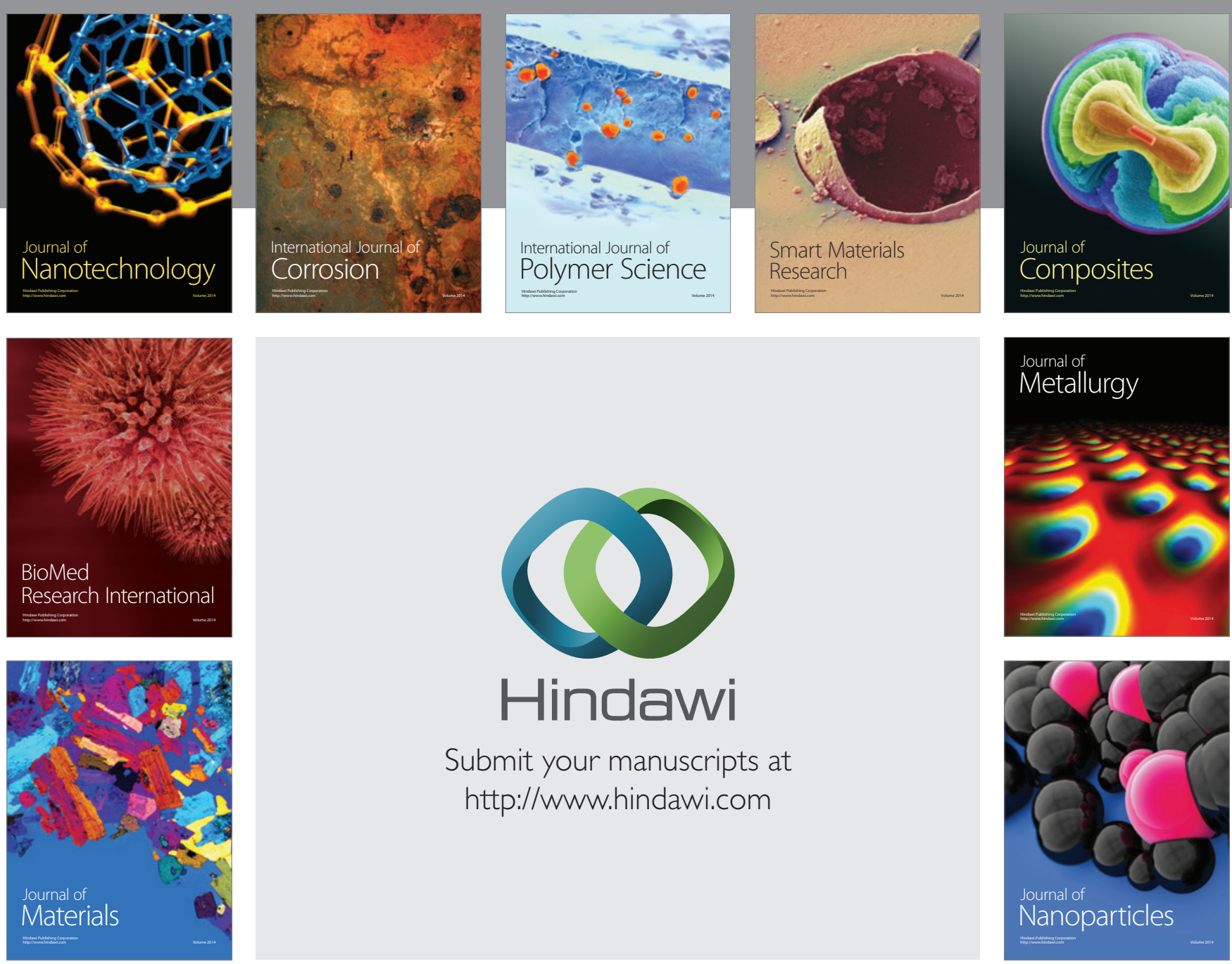

Submit your manuscripts at http://www.hindawi.com
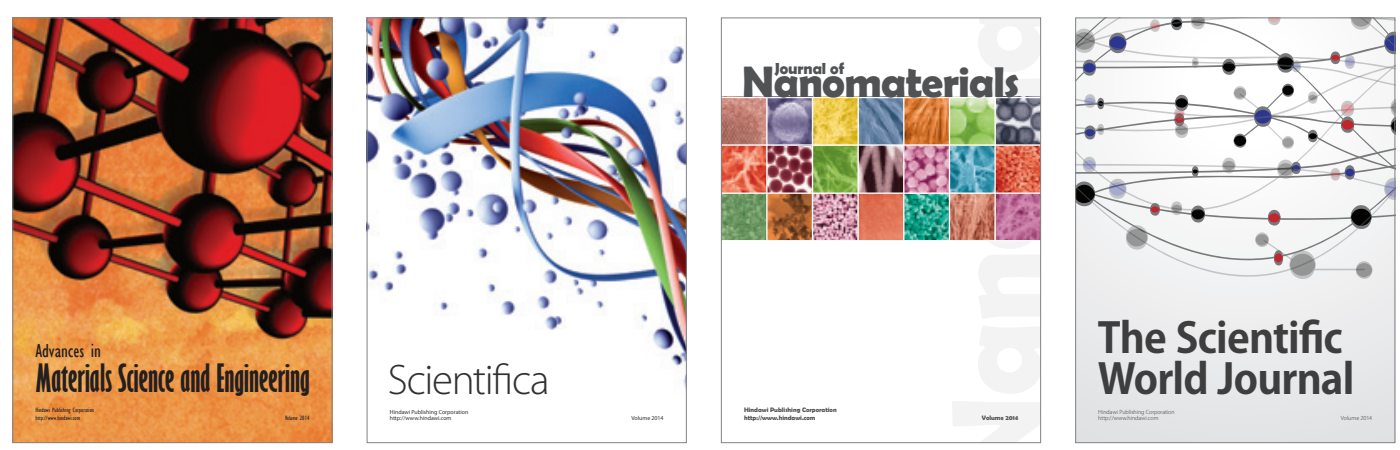

\section{The Scientific World Journal}
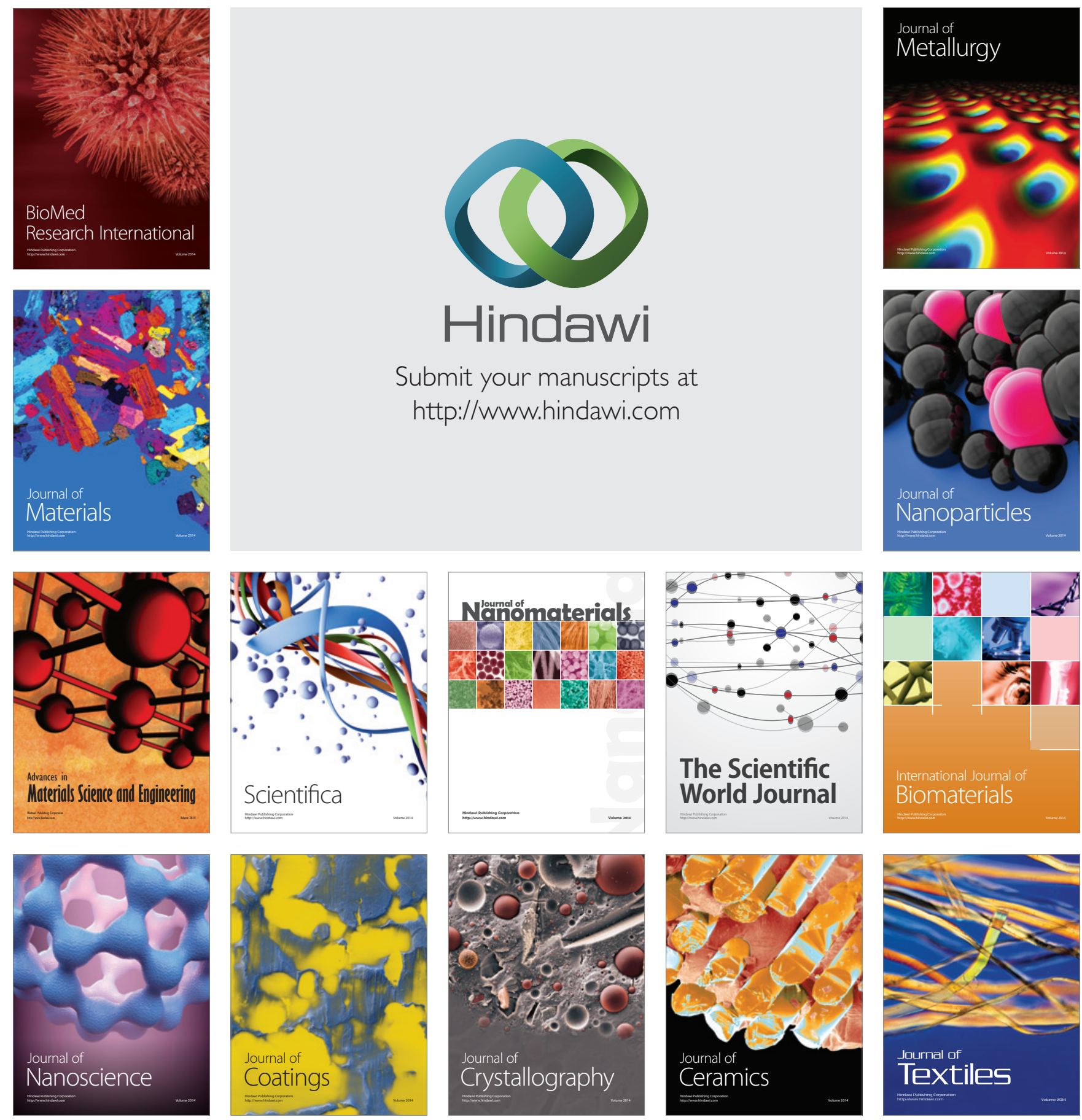